\title{
Splanchnic Vein Thrombosis - an Uncommon Complication after Laparoscopic Sleeve Gastrectomy
}

\author{
Tanja Carli Tadeja Pintar \\ Abdominal Surgery, UMC Ljubljana, Ljubljana, Slovenia
}

\section{Key Words}

Obesity · Laparoscopic sleeve gastrectomy · Thrombosis

\begin{abstract}
Background: Laparoscopic sleeve gastrectomy (LSG) is an innovative and relatively safe surgical approach for weight reduction in morbidly obese people. Splanchnic vein thrombosis (SVT) is an extremely rare complication of LSG and, if not recognized, carries a high mortality rate. This paper highlights a potentially lethal condition of SVT after LSG. Case Report: A 37-year-old morbidly obese woman was referred to our institution for LSG. Three weeks after the intervention, she was readmitted with abdominal pain, vomiting, nausea, diarrhea, and fever with positive family anamnesis to viral disease. Abdominal X-ray as well as utrasonography were both normal, and no X-ray contrast medium leakage was observed. One week later, she was readmitted with septic condition. An abdominal computed tomography scan diagnosed lienal vein thrombosis along its whole length and partial thrombosis of the superior mesenteric vein. Conclusion: SVT presents very heterogeneously, which makes it extremely challenging to diagnose and to make an appropriate treatment decision. With regard to the high prevalence of obesity and the increasing frequency of LSG, prompt diagnosis and management are crucial.

\section{Introduction}

Morbid obesity, defined as a BMI $\geq 40 \mathrm{~kg} / \mathrm{m}^{2}$ or a BMI $\geq 35 \mathrm{~kg} / \mathrm{m}^{2}$ in the presence of highrisk comorbid conditions [1], is one of the leading health problems worldwide. It increases the risk of type 2 diabetes, hypertension, cardiovascular disease, dyslipidemia, arthritis, 
Carli and Pintar: Splanchnic Vein Thrombosis - an Uncommon Complication after

Laparoscopic Sleeve Gastrectomy

nonalcoholic steatohepatitis, gallbladder disease, sleep apnea syndrome, and several types of cancer [2].

Laparoscopic sleeve gastrectomy (LSG) is rapidly gaining momentum in bariatric surgery, having the advantages of being less complex than other bariatric procedures and being associated with less malnutritive effects as well as comparable weight loss and resolution of comorbidities as Roux-en-Y gastric bypass [3].

Commonly reported complications of LSG include staple-line leak, respiratory insufficiency, pulmonary embolism, hemorrhage, stricture, and splenic injury [4]. Splanchnic vein thrombosis (SVT) is an unusual complication of LSG, which occurs in approximately $1 \%$ of cases $[5,6]$, and is, to our knowledge, described in only two case reports $[7,8]$. The aim of this paper is to present a unique case of SVT after SLG in a morbidly obese woman which was complicated by septic condition.

\section{Case Report}

A 37 -year-old woman with a baseline BMI of $50.2 \mathrm{~kg} / \mathrm{m}^{2}$ and preoperative BMI $42.2 \mathrm{~kg} / \mathrm{m}^{2}$ as well as comorbidities (arterial hypertension, chronic obstructive pulmonary disease, dilatative cardiomyopathy, status post cholecystectomy) and amoxicillin-induced hypersensitivity was referred to our institution for LSG.

The standard procedure lasted $45 \mathrm{~min}$ under an insufflation pressure of $14 \mathrm{~mm} \mathrm{Hg}$, and the patient tolerated it well. A subtherapeutic dose of 9,500 IU anti-Xa/1,0 ml was used to prevent thromboembolic episodes. The postoperative course was uncomplicated, and the patient was discharged on the 4th postoperative day with structured instructions for postoperative food, proton pump inhibitors (PPI), polyvitamin supplements, and physical activity recommendations.

Three weeks later, she was readmitted due to the diffuse and cramp-like abdominal pain, vomiting, nausea, diarrhea, and fever with positive family history to acute abdominal viral disease. Physical examination revealed afebrile, distended, soft abdomen painless to palpation, absence of organomegaly or other masses, presence of bowel sounds, and healed trocar incision sites per primam. Complete blood count revealed increases in C-reactive protein (CRP) as well as in creatinine, urea, and aminotransferases (AST, ALT). Abdominal X-ray and ultrasonography (US) were both normal, and no X-ray contrast medium leakage was observed. The patient was treated with intravenous glucosaline infusions, analgetics (paracetamol), PPI (omeprazol), and antibiotic (ertapenem) in standard dosage of $1 \mathrm{~g} /$ day. In the following days, she remained asymptomatic and afebrile, with no other complications, and was discharged on hospital day 3.

One week later she represented to our facility with septic condition. On admission, abdominal computed tomography (CT) scan and US revealed lienal vein thrombosis along its whole length, partial thrombosis of the superior mesenteric vein (SMV), several hypoechoic lesions, suggestive of hepatic abscesses - especially the one in the IVb segment $(19 \times 16 \mathrm{~mm}$ in diameter $)$-, and splenomegaly with small hypoechoic lesions, suggestive of infarcts. Thoracic CT scan was normal, while the echography showed earlier signs of hyperthrophic heart and minimal pleural effusion. The patient was immediately heparinized with low-molecularweight heparine - Fraxiparine ${ }^{\circledR} 5700$ anti-Xa IU/0.6 ml s.c. / $12 \mathrm{~h}$ and further on with Fraxiparine 7600 anti-Xa IU/0.8 $\mathrm{ml} \mathrm{s.c.} \mathrm{/} 12 \mathrm{~h}$. Also the antibiotics imipenem and cilastatin were given followed by solved amoxicillin p.o., according to a local hospital protocol. Control US revealed patent vena portae in its distal two-thirds, but, on contrary, the distal part of SMV and Vena lienalis could not be assessed. In the following days, clinical examination as well as laboratory tests improved, and the patient was discharged on hospital day 13.

One week later, she was readmitted again with septic condition. Urgent abdominal CT scan showed small hepatic abscess $(6 \times 4 \mathrm{~mm}$ in diameter $)$ in the IVb segment and several similar hepatic hypodense lesions, all of which were smaller than in the previously taken CT scan. Vena lienalis was thrombosed through its whole length, in contrast to patent Vena portae and SMV. US-guided percutaneous drainage procedure was performed to treat the largest hepatic abscess. The patient was treated with Fraxiparine 9500 anti-Xa IU $/ 2 \times 1 \mathrm{ml}$ s.c. $/ 12 \mathrm{~h}$, antibiotics piperacillin (Tazocine ${ }^{\circledR} 4.5 \mathrm{~g} / 8 \mathrm{~h}$ ) and amoxicillin (Amoxiclav $^{\circledR} 1.2 \mathrm{~g} / 8 \mathrm{~h}$ ), antiulcer drug (PPI, pantoprazol) and intravenous glucosaline infusions with polyvalent vitamin formula. 
Carli and Pintar: Splanchnic Vein Thrombosis - an Uncommon Complication after Laparoscopic Sleeve Gastrectomy

On hospital day 11, she was discharged, but re-administered the day after due to the short episode of hematemesis, for which 4 units of packed red cells were transfused. After several administrations to ambulant antithrombotics and, finally, following the recommended regime with Fraxiparine 9500 anti-Xa IU/1 ml s.c. / $12 \mathrm{~h}$, genital and gastrointestinal bleeding stopped. Gastroscopy showed no traces of blood down to the lower portion of the duodenum, and neither were any potential sources of bleeding detected. Due to clinical suspicion of septic emboli, a transthoracic echocardiogram (TEE) was performed, but no evidence for emboli was found. However, upon consultation with the infectiologists, new antibiotic therapy based on combination of cephalosporine and metronidazole was introduced with subsequent gradual decline in inflammatory markers. Also, analgetics as well as infusion solution of alanylglutamine (Dipeptiven ${ }^{\circledR}$ ) and vitamin B12, along with multivitamin supplements, were administered. Control US showed a homogeneous and uniformly enlarged spleen (17 cm in diameter) and complete recanalization in the splanchnic system. The patient was discharged 13 days later in stable condition. An excellent late postoperative course could be observed, with a 5-month weight loss of $40 \mathrm{~kg}$ (over $70 \mathrm{EWL} \%$ ), no metabolic complications, good physical performance, and improvement of muscular length and lean body mass detected in Tanita ${ }^{\mathrm{TM}}$ measurement system of body impedance.

\section{Discussion}

SVT is an unusual manifestation of venous thromboembolism which involves one or more abdominal veins (portal, splenic, mesenteric and supra-hepatic veins) [9]. According to the literature, it may be associated with different underlying disorders, either local (abdominal malignancy, liver cirrhosis, pancreatitis, intraabdominal inflammation, surgery), is systemic (myeloproliferative neoplasms, oral contraceptives, increased factor VIII level, hyperhomocysteinemia, prothrombin gene mutation, protein C deficiency) or idiopathic [10].

In patients undergoing bariatric surgery, metabolic syndrome, chronic inflammation, venous stasis, and intraoperative manipulation of splanchnic vasculature are among the suggested causative factors for SVT [11].

The mechanism by which laparoscopic surgery increases the risk of development of splanchnic vessel thrombosis remains unclear [12-17]. However, the main factor that is adversely affected during laparoscopy is venous stasis [18]. Insufflating the abdomen with pressures greater than $14 \mathrm{~mm} \mathrm{Hg}$ have been reported to reduce the portal blood flow by up to $53 \%[19,20]$. Insufflating with $\mathrm{CO}_{2}$ may also contribute to reduced intraoperative splanchnic blood flow [21] by causing the release of vasopressin [16]. In addition, mesenteric vasoconstriction results from hypercapnia related to intraperitoneal $\mathrm{CO}_{2}$ absorption, as described in experimental animal models $[16,22]$. Other factors that favor thrombosis by interrupting flow include the reverse Trendelenburg position [23] due to compressive effect of abdominal viscera on the iliac veins [18]. In our patient, a standardized $14 \mathrm{~mm} \mathrm{Hg} \mathrm{CO}$ insufflation in combination with reverse Trendelenburg position was utilized. Accuracy of surgical technique, reduction of the duration of surgery, and ideal anesthetic protocol using sugammadex for prompt reversal of neuromuscular block all reduce the risk for SVT [24].

Intraoperative surgical manipulation may damage the splanchnic endothelium and lead to local thrombus formation that may then propagate throughout the portal venous system. This may be particularly true for laparoscopic splenectomy, in which ligation of the splenic vein causes endothelial damage in proximity to the portal vein [25], but not in our case. Many other procedures may also lead to some manipulation of the splanchnic vasculature, but in our opinion intraoperative surgical manipulation during sleeve gastrectomy which only involves transection of the short gastric veins is less likely to be the cause of SVT, provided that surgical technique is good and no intraoperative bleeding occurs.

Metabolic syndrome resulting in a hypercoagulable state may also contribute to postoperative venous thrombosis in our patient as obesity promotes thrombosis through a variety of mechanisms, including adipocytokines, depression of the fibrinolytic cascade and augmen- 
Carli and Pintar: Splanchnic Vein Thrombosis - an Uncommon Complication after Laparoscopic Sleeve Gastrectomy

tation of the coagulation cascade, increased inflammation, and endothelial dysfunction [23, 26].

SVT occurs with broad spectrum of clinical presentations, ranging from incidental findings in an asymptomatic patient to concomitant presence of extensive thrombosis and gastrointestinal bleeding. Clinical presentations of acute SVT are most commonly seen approximately 2 weeks postoperatively. Different underlying factors can trigger SVT - among them also infectious diseases. Like in our case, patients initially complain of nonfocal abdominal pain, nausea, vomiting, and low-grade fever [27]. Laboratory values are typically within normal limits, but leukocytosis and mild elevation of liver function tests can also be seen [7], the former also noted in our patient. However, this presentation is nonspecific, especially since vague abdominal pain, nausea, and vomiting can also be seen for a few weeks in the normal postoperative course of sleeve gastrectomy [7]. Nonetheless, as shown in our case, SVT is a potentially life-threatening condition, in which an extensive thrombosis and subsequent intestinal infarction led to ascites, gastroesophageal hemorrhage, and septic shock.

For obese patients, CT is an excellent means to evaluate gastrointestinal or nonspecific complaints in the postoperative course of surgical procedures [26, 29]. Invasive procedures are usually not needed, unless there is a high degree of suspicion [30]. In our case, gastroscopy was performed for detection of any potential source of bleeding and exclusion of the presence of peptic ulcer and gastroesophageal varices. Although US is readily available and expedient, it has the lowest specificity for detection of SVT of available imaging techniques [31] and is best used to document restoration of venous flow in a patient with known SVT. Magnetic resonance imaging is highly sensitive and specific for SVT but not widely available [32]. In the absence of major contraindications, anticoagulant therapy is generally recommended for all patients presenting with acute symptomatic SVT, starting with either low-molecular weight or unfractionated heparin and continuing with the vitamin K antagonists in most patients [33, 34]. We began to treat SVT with high therapeutic dose of low-molecular-weight heparin, which was subsequently shifted to half a dose because of bleeding and then replaced by warfarin. Along with specific antibiotic therapy, the patient recovered completely. The recommended duration of anticoagulation treatment is 6-12 months [25]. Some studies suggested that the risk of thromboembolism following bariatric surgery extended long after discharge from the hospital, and prophylaxis should therefore be continued for several weeks into the postoperative period [35]. However, this aggressive approach, which could also theoretically lower the risk of SVT, is not considered standard [36].

\section{Conclusion}

SVT is an uncommon complication of sleeve gastrectomy in morbidly obese patients. Clinical presentations are heterogeneous ranging from asymptomatic findings to bowel infarction and sepsis. As this condition is potentially life-threatening, the diagnosis should be prompt. For acute symptomatic SVT in patients without major contraindications, prompt anticoagulant therapy in therapeutic dose is the current standard. Also, doses of all medications are suggested to be calculated to actual BMI/body weight, especially those with fatty tissue-wide distribution. However, recommendations are derived only from observational studies, and further research is needed for fully delineation on the acute management of SVT after laparoscopic surgery.

\section{Disclosure Statement}

No reported conflicts of interest. 
Carli and Pintar: Splanchnic Vein Thrombosis - an Uncommon Complication after Laparoscopic Sleeve Gastrectomy

\section{References}

1 Buchwald H: Bariatric surgery for morbid obesity: health implications for patients, health professionals, and third-party payers. Surg Obes Relat Dis 2005;1:371-381.

2 James WPT, Rigby N, Leach R: The obesity epidemic, metabolic syndrome and future prevention strategies. Eur J Cardiovasc Prev Rehab 2004;11:3-8.

3 Aurora AR, Khaitan L, Saber AA: Sleeve gastrectomy and the risk of leak: a systematic analysis of 4,888 patients. Surg Endosc 2012;26:1509-1515.

4 Gumbs AA, Gagner M, Dakin G, Pomp A: Sleeve gastrectomy for morbid obesity. Obes Surg 2007;17:962-969.

5 Alsina E, Ruiz-Tovar J, Alpera MR, Ruiz-García JG, Lopez-Perez ME, Ramon-Sanchez JF, et al: Incidence of deep vein thrombosis and thrombosis of the portal-mesenteric axis after laparoscopic sleeve gastrectomy. J Laparoendosc Adv Surg Tech A 2014;24:601-605.

6 Salinas J, Barros D, Salgado N, Viscido G, Funke R, Pérez G, et al: Portomesenteric vein thrombosis after laparoscopic sleeve gastrectomy. Surg Endosc 2014;28:1083-1089.

7 Rosenberg JM, Tedesco M, Yao DC, Eisenberg D: Portal vein thrombosis following laparoscopic sleeve gastrectomy for morbid obesity. JSLS 2012;16:639-643.

8 Flaherty DC, Ichter Z, Rideman ED, Mical E, Berwick A, Fakulujo A, Neff M: Superior mesenteric vein thrombosis after laparoscopic sleeve gastrectomy: a case report. www.rowan.edu/som/education/graduate medical/documents/flaherty_Poster.pdf (last accessed March 23, 2016).

-9 Riva N, Donadini MP, Dentali F, Squizzato A, Ageno W: Clinical approach to splanchnic vein thrombosis: Risk factors and treatment. Thromb Res 2012;130:1-3.

$\checkmark 10$ Ponziani FR, Zocco MA, Campanale C, Rinninella E, Tortora A, Di Maurizio L, et al: Portal vein thrombosis: insight into physiopathology, diagnosis, and treatment. World J Gastroenterol 2010;16:143-155.

-11 Zocco MA, Di Stasio E, De Cristofaro R, Novi M, Ainora ME, Ponziani F, et al: Thrombotic risk factors in patients with liver cirrhosis: correlation with MELD scoring system and portal vein thrombosis development. J Hepatol 2009;51:682-689.

-12 Stemberg A, Alfici R, Bronek S: Laparoscopic surgery and splanchnic vessels thrombosis. J Laparoendosc Adv Surg Tech A 1998;8:65-68.

13 Abdelrazeq AS, Dwaik MA, Aldoori MI, Lund JN, Leveson SH: Laparoscopy-associated portal vein thrombosis: description of an evolving clinical syndrome. J Laparoendosc Adv Surg Tech A 2006;16:9-14.

14 Poultsides GA, Lewis WC, Feld R, et al: Portal vein thrombosis after laparoscopic colectomy: thrombolytic therapy via the superior mesenteric vein. Am Surg 2005;71:856-860.

15 Targarona E: Portal vein thrombosis after laparoscopic splenectomy: the size of the risk. Surg Innov 2008;15: 266-270.

16 Denne J, Kowalski C: Portal vein thrombosis after laparoscopic gastric bypass. Obes Surg 2005;15:886-889.

-17 Paye F, Faitot F, Rossol A: Trombose de la veine porte par migration intragastrique d'un anneau gastrique. Gastroenterol Clin Biol 2009;2009:203-205.

18 Nguyen NT, Wolfe BM: The physiologic effects of pneumoperitoneum in the morbidly obese. Ann Surg 2005; 241:219-226.

19 Noh KW, , Wolfsen HC, Bridges MD, Hinder RA: Mesenteric venous thrombosis following laparoscopic antireflux surgery. Dig Dis Sci 2007;52:273-275.

20 Jakimowicz J, Stultiens G, Smulders F: Laparoscopic insufflation of the abdomen reduces portal venous flow. Surg Endosc 1998;12:129-132.

-21 James AW, Rabl C, Westphalen AC, Fogarty PF, Posselt AM, Campos GM: Portomesenteric venous thrombosis after laparoscopic surgery. Arch Surg 2009;144:520-526.

22 Preventza QA, Habib FA, Young Shun C, Penny D, Oppat W, Mittal V: Portal vein thrombosis: an unusual complication of laparoscopic cholecystectomy. JSLS 2005;9:87-90.

23 Darvall KA, Sam RC, Silverman SH, Bradbury AW, Adam DJ: Obesity and thrombosis. Eur J Vasc Endovasc Surg 2007;33:223-233.

-24 Rahe-Meyer N, Fennema H, Schulman S, Klimscha W, Przemeck M, Blobner M, et al: Effect of reversal of neuromuscular blockade with sugammadex versus usual care on bleeding risk in a randomized study of surgical patients. Anesthesiology 2014;121:969-977.

-25 James AW, Rabl C, Westphalen AC, Fogarty PF, Posselt AM, Campos GM: Portomesenteric venous thrombosis after laparoscopic surgery a systematic literature review. Arch Surg 2009;144:520-526.

26 Goitein D, Matter I, Raziel A, Keidar A, Hazzan D, Rimon U, et al: Portomesenteric thrombosis following laparoscopic bariatric surgery. JAMA Surg 2013;148:340-346.

27 Parikh S, Shah R, Kapoor P: Portal vein thrombosis. Am J Med 2010;123:111-119.

28 Swartz DE, Felix EL: Acute mesenteric venous thrombosis following laparoscopic Roux-en-Y gastric bypass JSLS 2004;8:165-169.

29 Johnson CM, de la Torre RA, Scott JS, Johansen T: Mesenteric venous thrombosis after laparoscopic Roux-en-Y gastric bypass.. Surg Obes Relat Dis 2005;1:580-582; discussion 582-583

30 Franco J, del Castillo J, Veláquez A, Martinez J: Portal thrombosis after laparoscopic bariatric surgery. Description of 3 cases and systematic literature rewiew. BMI 2011;1:1-5. http://bmilatina.com/index.php/ bmi/article/viewFile/32/pdf_1 (last accessed March 23, 21106). 
Carli and Pintar: Splanchnic Vein Thrombosis - an Uncommon Complication after Laparoscopic Sleeve Gastrectomy

31 Pieters PC, Miller WJ, DeMeo JH: Evaluation of the portal venous system: complementary roles of invasive and noninvasive imaging strategies. Radiographics 1997;17:879-895.

-32 Haddad MC, Clark DC, Sharif HS, al Shahed M, Aideyan O, Sammak BM: MR, CT, and ultrasonography of splanchnic venous thrombosis. Gastrointest Radiol 1992;17:34-40.

-33 De Franchis R; on behalf of the Baveno V Faculty: Revising consensus in portal hypertension: report of the Baveno $\mathrm{V}$ consensus workshop on methodology of diagnosis and therapy in portal hypertension. J Hepatol 2010;53:762-768.

34 Kearon C, Akl EA, Comerota AJ, Prandoni P, Bounameaux H, Goldhaber SZ, et al: American College of Chest Physicians. Antithrombotic therapy for VTE disease: Antithrombotic Therapy and Prevention of Thrombosis, 9th ed: American College of Chest Physicians Evidence-Based Clinical Practice Guidelines. Chest 2012;141(2 Suppl):e419S-94S. Erratum in Chest 2012;142:1698-1704.

-35 Steele KE, Schweitzer MA, Prokopowicz G, Shore AD, Eaton LC, Lidor AO, Makary MA, Clark J, Magnuson TH: The long-term risk of venous thromboembolism following bariatric surgery. Obes Surg 2011;21:1371-1376.

-36 Clinical Issues Committee of the American Society for Metabolic and Bariatric Surgery: Prophylactic measures to reduce the risk of venous thromboembolism in bariatric surgery patients. Surg Obes Relat Dis 2007;3: 494-495. 